\title{
Competitividad, desarrollo e ingeniería: algunas definiciones y reflexiones
}

\author{
Bernardo J. Pérez-Castaño*s \\ * Escuela de Ingeniería Industrial y Estadística, Universidad del Valle, Cali, Colombia \\ §e-mail: beperez@univalle.edu.co
}

(Recibido: Octubre 20 de 2006 - Aceptado: Junio 15 de 2007)

\begin{abstract}
Resumen
Este artículo está dirigido a quienes proponen o desarrollan modelos, procesos y productos de interés en cualquier organización, buscando mejorar su competitividad, para generar más empleo y contribuir a la formación y utilización de capacidades humanas, satisfaciendo las necesidades existenciales y esenciales de las personas. Este artículo no está dirigido a especialistas en el tema, ni es un manual de métodos y procedimientos para mejorar la competitividad. Es una introducción básica al tema, escrita en lenguaje sencillo para mostrar, amena y claramente, aspectos relacionados con la competitividad, el desarrollo y la ingeniería, destacando sus interrelaciones. Inicialmente, se introduce, para cada término, la definición tomada del Diccionario de la Real Academia de la Lengua Española y posteriormente, para aportar más elementos de juicio, se presentan los conceptos de diversos autores con trayectoria en el tema. Finalmente, se presentan algunas reflexiones sobre la importancia del papel que desempeña la ingeniería en el desarrollo y la competitividad.
\end{abstract}

Palabras clave: Competitividad, Desarrollo, Desarrollo humano, Ingeniería competitiva, Tecnología.

\section{Competitiveness, development, and engineering: some definitions and reflections}

\begin{abstract}
This paper is addressed to those who propose or develop models, processes, and products of interest to any organization, seeking the improvement of its competitiveness, to create more jobs and contribute to the education and usage of human abilities, whereby fulfilling people's life and basic needs. This paper is neither addressed to experts in the subject nor is a handbook of methods and procedures for improving competitiveness. It is a basic introduction to the subject, written in a simple language, to discuss, in a clear and pleasant way, some issues related to competitiveness, development, and engineering, underlining their mutual relationship. Firstly, the definition of each term (taken from the Royal Academy of the Spanish Language Dictionary) is introduced. To gather more judgement elements, these definitions are followed by a discussion of some concepts given by various authorative experts. Lastly, some reflections are given on the important role played by engineering in development and competitiveness.
\end{abstract}

Keywords: Competitiveness, Development, Human development, Competitive engineering, Technology. 


\section{Introducción}

Este artículo está dirigido a quienes proponen o desarrollan técnicas, procedimientos, modelos, procesos, productos y servicios, para su aplicación tanto en empresas productivas como en organizaciones sin ánimo de lucro económico, buscando mejorar su competitividad, en pro de una mayor generación de empleo y, en general, de contribuir a la formación y utilización de las capacidades humanas para satisfacer tanto las necesidades existenciales como las necesidades esenciales de las personas.

Este artículo no está dirigido a especialistas en el tema, ni es un manual de métodos y procedimientos para mejorar la competitividad de las empresas, regiones o naciones. Es una introducción básica al tema, escrita en un lenguaje sencillo para mostrar, de una manera amena y clara, algunos aspectos relacionados con la competitividad, el desarrollo y la ingeniería, destacando sus interrelaciones y el papel que juega la ingeniería para lograr la competitividad y el desarrollo.

Ohmae (2005) afirma que la terminología es siempre una ciencia inexacta. Cada término es un colador lingüístico. Sin embargo, es parte de la naturaleza humana buscar una definición para cada concepto. Conservando esta línea, para cada término, se presenta inicialmente la definición tomada del Diccionario de la Real Academia de la Lengua Española y posteriormente, para aportar más elementos de juicio, se discuten los conceptos de diversos autores con trayectoria en el tema.

\section{Competitividad y desarrollo}

En su época, Aristóteles advirtió que las sociedades no deben juzgarse simplemente por patrones tales como el ingreso y la riqueza, los cuales no se buscan por si mismos, sino que se desean como medios para alcanzar otros objetivos (Morris, 1997). De la misma manera, la competitividad, simbolizada por una mayor riqueza, es un medio para alcanzar el desarrollo, simbolizado por un mayor bienestar.

Cabe aclarar que, aunque a lo largo de este texto, el concepto de competitividad esté en el marco de una nación o región, en general, es perfectamente asimilable a cualquier tipo de organización e inclusive a personas.

En cuanto al desarrollo, es preciso tener en cuenta que se refiere a las personas, a la sociedad, no a los objetos. El Programa de las Naciones Unidas para el Desarrollo (UNDP, 1990), sostiene que el objetivo básico del desarrollo es crear un ambiente propicio para que los seres humanos disfruten de una vida prolongada, saludable y creativa. Esta puede parecer una verdad obvia, aunque con frecuencia se olvida debido a la preocupación inmediata de acumular bienes de consumo y riqueza financiera.

\subsection{Competitividad}

- Competitividad: Capacidad de competir. Rivalidad para la consecución de un fin. (RAE, 2001). Michael Porter, Profesor de la Universidad de Harvard, es uno de los autores modernos que más ha investigado y escrito sobre competitividad. Para Porter (1982, 1983, 1985, 1988, 1990), la principal meta económica de una nación es producir un alto y creciente nivel de vida para sus ciudadanos. La capacidad de conseguirlo depende no de la amorfa noción de competitividad, sino la productividad con que se empleen los recursos de una nación (trabajo y capital). La productividad es el valor del producto generado por unidad de trabajo o capital. Depende tanto de la calidad y de las características de los productos (lo que determina los precios a que pueden venderse) y la eficiencia con que se producen.

Otro concepto de competitividad muy reconocido es el del IMD (Institute for Management Development) que desarrolló un modelo y una metodología (IMD, 1996) para medir la competitividad de las naciones, los cuales se vienen utilizando desde 1989 y cuyos resultados son tenidos en cuenta por académicos, gobernantes y empresarios como un insumo importante para la definición de sus estrategias. Para el IMD, competitividad es la capacidad de un país de crear valor agregado y consecuentemente incrementar la riqueza nacional mediante la gestión de sus activos y procesos, de su atracción y agresividad, de su globalidad y proximidad, y a 
través de la integración de esas interrelaciones en un modelo económico y social.

En la Constitución Nacional de Colombia de 1991, actualmente vigente, se encuentra un concepto que refleja una característica importante de la competitividad, como es la flexibilidad o capacidad de ajustarse a las condiciones predominantes.

Landau, reconocido economista estadounidense, presenta un concepto más amplio y de contenido social más explicito. Landau (1991) dice que se debería entender por competitividad la capacidad de mantener, en una economía global, un crecimiento de la calidad de vida de la población y una justicia distributiva socialmente aceptables, al mismo tiempo que se provee eficientemente de empleo a quienes desean trabajar. Éste debería ser el principal objetivo de las políticas públicas. Además, todo ello se debería hacer sin reducir el potencial aumento en la calidad de vida de las generaciones futuras, lo cual restringe el endeudamiento exterior, o el uso excesivo de los impuestos $\mathrm{u}$ obligaciones de pago futuros, como pago por un mayor nivel de vida de las generaciones presentes.

\subsection{Desarrollo}

- Desarrollo: Evolución progresiva de una economía hacia mejores niveles de vida. (RAE, 2001).

Para Ackoff (1991) el desarrollo no es fácil de definir. Desarrollarse es el deseo y la capacidad de satisfacer las necesidades y deseos legítimos propios, así como los de los demás. Una necesidad o deseo legítimo es aquel que, cuando se satisface, no impide a los demás la posibilidad de satisfacer sus necesidades y deseos legítimos.

De acuerdo con Drucker (1992, 1994), el desarrollo es inteligente, pensante, visionario, estratégico. Es consciente de sus posesiones, de sus faltantes y una sociedad desarrollada busca y encuentra el recurso; lo utiliza con propiedad. No toma nada por hecho. El desarrollo aprende del fracaso; no se limita a inculpar, retoma su historia, su pasado, evalúa lo que tiene y continúa su marcha.
Siguiendo a Ackoff(2003), el desarrollo constante exige el perfeccionamiento continuo de los medios que se emplean en la prosecución de los fines. Por lo tanto, en la búsqueda del desarrollo hay cuatro aspectos interactuantes: científicotecnológico, económico, ético-moral y estético.

\subsubsection{Aspecto científico-tecnológico del desarrollo}

El perfeccionamiento continuo de los medios contribuye al desarrollo, cuando es conseguido a través de los descubrimientos científicos y de su aplicación práctica mediante la tecnología, para lograr una mayor eficiencia.

Las instituciones educativas juegan un papel preponderante en este aspecto puesto que, una de sus funciones, es dar a conocer información sobre la disponibilidad y el uso de los medios recientemente desarrollados.

\subsubsection{Aspecto económico del desarrollo}

La abundancia de recursos contribuye al desarrollo, ya que los medios son cursos de acción que frecuentemente requieren el uso de recursos: equipo e inatalaciones, materiales, energía, servicios, dinero e información.

Las instituciones económicas de una sociedad juegan un papel preponderante en este aspecto puesto que una de sus funciones es perseguir esa abundancia. El estándar de vida es la medida más comúnmente utilizada para juzgar el grado hasta el cual han triunfado en ese objetivo.

“ Si una sociedad libre no puede ayudar a sus muchos pobres, no podrá salvar a sus pocos ricos".

(John F. Kennedy)

\subsubsection{Aspecto ético-moral del desarrollo}

Aumentar la capacidad de logro de los objetivos (tanto personales como grupales), reduciendo los conflictos (tanto internos como externos), contribuye al desarrollo, debido a que cuando se presentan simultáneamente dos necesidades o deseos, si la satisfacción de uno implica necesariamente la no satisfacción del otro, éstos están en conflicto. 
Las instituciones dedicadas a fomentar la ética y la moral, objetivos del bien, juegan un papel preponderante en este aspecto puesto que una de sus funciones es la reducción de esos conflictos.

\subsubsection{Aspecto estético del desarrollo}

Divertirse e inspirarse a partir de lo que se hace contribuye al desarrollo.

Este aspecto tiene que ver con las facetas creativas y recreativas de perseguir un ideal. La diversión, entendida como la satisfacción que se obtiene de lo que se hace, independientemente de por qué se hace. La recreación, entendida como proporcionar las pausas que refrescan la búsqueda del ideal de desarrollo y de otros que, por su misma naturaleza, son inalcanzables. La persecución constante de un ideal exige, además, la visión de algo más deseable que el estado actual aceptando sacrificios en el corto plazo para alcanzar un progreso en el largo plazo. Requiere además coraje no solo para perseguirlo sino también para admitir que se requiere ayuda para ello.

\subsubsection{Desarrollo y calidad de vida}

De acuerdo con Ackoff (2003), dos ingredientes esenciales de la calidad de vida son la satisfacción obtenida de lo que se hace, independientemente de por qué se hace, y la satisfacción que se obtiene del comportamiento que impulsa hacia ideales significativos.

La calidad de vida puede ser alcanzada dependiendo de la cantidad y el tipo de recursos disponibles y del nivel de desarrollo que se tenga, convirtiendo así al desarrollo en un potencial para mejorar la calidad de vida actualmente alcanzada.

El estándar de vida se determina con lo que se tiene. La calidad de vida se determina con lo que se puede hacer a partir de lo que se tiene. En este sentido puede decirse que el estándar de vida se asocia con el crecimiento y la calidad de vida con el desarrollo.

Por lo tanto, la calidad de vida es fundamentalmente una cuestión de estética; el estándar de vida es fundamentalmente una cuestión de economía.

\subsubsection{Concepto de desarrollo humano}

Mahbub ul Haq (1995), destacado economista paquistaní, y Amartya Sen (1973, 1976 2000), célebre economista bengalí, Premio Nóbel de Economía en 1998, fueron precursores de las teorías de desarrollo humano, con base en las cuales el PNUD (1990) diseñó e implementó una metodología para medir el índice de desarrollo humano (IDH) de un país, tomando como base lo siguiente:

- El desarrollo humano es un proceso en el cual se amplían las oportunidades del ser humano. En principio, estas oportunidades pueden ser infinitas y cambiar con el tiempo. Sin embargo, a todos los niveles del desarrollo, las tres más esenciales son: disfrutar de una vida prolongada y saludable, adquirir conocimientos y tener acceso a los recursos necesarios para lograr un nivel de vida decente.

- El ser humano requiere poseer dichas oportunidades esenciales para que muchas otras alternativas sean accesibles de tal manera que logre un desarrollo humano completo. Oportunidades altamente valoradas por muchas personas, que van desde la libertad política, económica y social, hasta la posibilidad de ser creativo y productivo, respetarse a sí mismo y disfrutar de la garantía de derechos humanos.

- El desarrollo humano tiene dos aspectos: la formación de capacidades humanas tales como un mejor estado de salud, conocimientos y destrezas y el uso que la gente hace de las capacidades adquiridas para el descanso, la producción o las actividades culturales, sociales y políticas. Si el desarrollo humano no consigue equilibrar estos dos aspectos, pude generarse una considerable frustración humana.

- Según este concepto de desarrollo humano, es obvio que el ingreso es sólo una de las oportunidades que la gente desearía tener, aunque ciertamente muy importante. Pero la vida no sólo se reduce a eso. Por lo tanto, el desarrollo debe abarcar más que la expresión de la riqueza y los ingresos. Su objetivo central debe ser el ser humano. 


\subsection{Medición de la competitividad y del desarrollo}

En la medición de la competitividad de las naciones, se acostumbra emplear diferentes tipos de datos para medir variables cuantificables y aspectos cualitativos separadamente. Para lo cuantitativo, se acude a indicadores estadísticos que provienen de organizaciones nacionales, internacionales y regionales. Las variables cuantitativas representan una parte sustancial del valor en el escalafón general y determinan el ordenamiento general de competitividad. Lo cualitativo proviene de encuestas de opinión empresarial con un peso de menor valor en el ordenamiento general (Monitor Company, 1994 y 1996).

En general, para medir y controlar cualquier sistema, lo ideal sería incluir muchas variables para obtener un panorama lo más amplio posible, especialmente cuando se trata de sistemas complejos. Sin embargo, hay casos en que el exceso de indicadores podría crear una imagen confusa. Por ello, cuando esto ocurre, es conveniente centrar la medición en unos pocos elementos vitales, lo cual de paso facilita la focalización de las políticas; esto ocurre con el IDH.

En la medición del IDH de los países, el PNUD emplea solamente datos para medir variables cuantificables y acude a indicadores estadísticos que provienen de organizaciones nacionales, internacionales y regionales. Los cuadros del IDH proporcionan una evaluación general de los logros alcanzados por los países en distintas áreas del desarrollo humano.

\subsubsection{Medición de la competitividad}

Anuario Mundial de Competitividad (World Competitiveness Yearbook, WCY): el IMD publica desde 1989 el WCY, un estudio anual sobre la competitividad de los países, en el que se analiza y hace un ordenamiento de la habilidad de los países para proveer un entorno de apoyo a la competitividad de las empresas. El trabajo parte de reconocer que la competitividad de un país no puede reducirse solamente al PIB y la productividad, teniendo en cuenta las dimensiones políticas, sociales y culturales en las que desarrollan su actividad las empresas. Lo anterior plantea a las naciones la necesidad de proveer un entorno con la estructura, instituciones y políticas más eficientes que promuevan la competitividad de las empresas.

El WCY proporciona un cubrimiento de 60 economías nacionales y regionales. Como resultado de una extensa investigación y del recurso a la literatura económica, fuentes nacionales, regionales e internacionales, y la retroalimentación de la comunidad empresarial, gobiernos y academia, el estudio considera 323 criterios de evaluación, agrupados en cuatro factores de competitividad: desempeño económico, eficiencia del gobierno, efíciencia empresarial e infraestructura.

En la preparación de este estudio, el IMD emplea indicadores estadísticos de organizaciones nacionales, internacionales y regionales que constituyen las variables cuantitativas usadas para determinar el ordenamiento general de competitividad, los cuales representan dos tercios del valor en el escalafón general. Los restantes criterios provienen de la encuesta de opinión empresarial, y conforman los datos cualitativos, cuyo peso en el ordenamiento general es de $1 / 3$. Para los 60 países considerados, se construye un escalafón en el que se presentan las economías de mayor a menor nivel de competitividad. La publicación del WCY tiene lugar cada año en el mes de mayo.

El Ministerio de Comercio Exterior de Colombia (MINCOMERCIO, 2006) elabora un documento de análisis sobre la posición de Colombia en este estudio.

Reporte Global de Competitividad (Global Competitiveness Report, GCR): el Foro Económico Mundial (WEF, 2006) publica anualmente el reporte global de competitividad (RGC) en el cual se evalúa el potencial de crecimiento económico sostenible de un grupo de más de 100 economías, se presenta una evaluación comparativa de sus debilidades y fortalezas, y se clasifican de acuerdo con dos enfoques diferentes pero complementarios en el análisis de la competitividad económica: índice de crecimiento 
de la competitividad (ICC), e indice de competitividad empresarial (ICE).

El primer enfoque (ICC) es liderado por Jeffrey Sachs, actualmente director del Earth Institute de la Universidad de Columbia, se basa en el concepto de competitividad global como el conjunto de instituciones y políticas económicas que sirven de apoyo a la existencia de altas tasas de crecimiento económico en el mediano plazo. Con base en fundamentos macroeconómicos teóricos y empíricos, el WEF calcula el ICC como la mejor estimación de las perspectivas de crecimiento durante los siguientes cinco años para las economías analizadas.

El segundo enfoque (ICE) corresponde al liderado por Michael Porter del Instituto de Estrategia y Competitividad de la Escuela de Administración de la Universidad de Harvard. El ICE hace uso de indicadores microeconómicos para la medición del conjunto de instituciones, estructuras de mercado y políticas económicas que sirven de base para el logro de altos niveles de prosperidad (esto es, la efectiva utilización que hace la economía de su acervo actual de recursos). De esta manera, el ICE evalúa el potencial productivo actual de las economías analizadas.

En conjunto, el ICC y el ICE presentan dos enfoques distintos pero complementarios con los que se exploran las fuentes de la competitividad nacional. Ambos combinan datos cuantitativos con datos cualitativos obtenidos de la aplicación de una encuesta de opinión (la cual permite derivar datos sobre una buena cantidad de aspectos de la economía de un país para los que no existen datos cuantitativos, tales como la eficiencia de las instituciones de gobierno, la sofisticación de las redes de abastecimiento local, o la naturaleza de las prácticas competitivas).

Recientemente el RGC incluye un nuevo índice de competitividad: el indice global de competitividad (IGC), el cual se diseñó con el objeto de unificar los dos índices actualmente utilizados. La publicación del RGC tiene lugar cada año en el mes de octubre.

El Ministerio de Comercio Exterior de Colombia (MINCOMERCIO, 2005) elabora un documento de análisis sobre la posición de Colombia en este estudio.

\subsubsection{Medición del desarrollo}

Como ya se mencionó, el PNUD diseñó el IDH como un indicador compuesto que mide los avances promedio de un país en función de las tres dimensiones básicas del desarrollo humano, a saber: vida larga y saludable, medida según la esperanza de vida al nacer; educación, medida por la tasa de alfabetización de adultos y la tasa bruta combinada de matriculación en enseñanza primaria, secundaria y terciaria; y nivel de vida digno, medido por el PIB per cápita (PPA en US\$). Este último se representa con el ingreso pero con la salvedad de que el indicador debe reflejar los rendimientos decrecientes al transformar el ingreso en capacidades humanas, dado que los individuos no necesitan recursos financieros en exceso para garantizar una vida decente. Para corroborar lo anterior, a manera de ejemplo, Colombia ocupa el puesto 69 en la clasificación del IDH (UNDP, 2005a) y el puesto 77 (UNDP, 2005b) en la de PIB per cápita, mientras que Argentina ocupa el 34 (UNDP, 2005a) y el 42 (UNDP, 2005b), respectivamente.

El IDH se construye con indicadores que en la actualidad están disponibles en todo el mundo y utiliza una metodología a la vez simple y transparente.

Sin embargo, el IDH tiene limitaciones puesto que omite oportunidades que la gente valora considerablemente, tales como, libertad, tanto económica como social y política, protección contra la violencia, inseguridad, discriminación, entre otras.

Adicionalmente, el PNUD (1990) definió los ocho objetivos de desarrollo del milenio para el año 2015, que abarcan desde la reducción de la pobreza extrema a la mitad hasta la detención de la propagación del VIH/SIDA y la consecución de la enseñanza primaria universal:

- erradicar la pobreza extrema y el hambre.

- lograr la enseñanza primaria universal.

- promover la igualdad entre los géneros y la autonomía de la mujer. 
- reducir la mortalidad infantil.

- mejorar la salud materna.

- combatir el VIH/SIDA, el paludismo y otras enfermedades.

- garantizar la sostenibilidad del medio ambiente.

- fomentar una asociación mundial para el desarrollo.

Estos objetivos constituyen un plan convenido por todas las naciones del mundo $\mathrm{y}$ todas las instituciones de desarrollo más importantes a nivel mundial. Los objetivos han galvanizado esfuerzos sin precedentes para ayudar a los más pobres del mundo.

Informe Mundial de Desarrollo Humano 2005 (UNDP, 2005): los cuadros de indicadores del desarrollo humano proporcionan una evaluación general de los logros alcanzados por los países en distintas áreas del desarrollo humano. Muchos de los indicadores de los objetivos de desarrollo del milenio están incluidos en estos cuadros.

Los cuadros principales están organizados por tema e incluyen información del universo de 193 países y regiones cubiertas: 191 estados miembros de las Naciones Unidas, más Hong Kong, China y los Territorios Palestinos Ocupados.

Los países se clasifican en cuatro formas: por nivel de desarrollo humano, por ingreso, por principales agrupaciones mundiales y por región. Estas designaciones no necesariamente expresan un juicio acerca de la etapa de desarrollo en que se encuentra un país o una zona determinada. El término país, tal como se utiliza en el texto y en los cuadros, también se refiere a territorios o zonas, según corresponda.

Clasificaciones según el desarrollo humano. Todos los países que se incluyen en el IDH se clasifican en tres grupos atendiendo a sus logros en desarrollo humano: desarrollo humano alto (con IDH igual o superior a 0.800 ), desarrollo humano medio (IDH de 0.500 a 0.799) y desarrollo humano bajo (IDH inferior a 0.500 ).

Clasificaciones según ingreso. Todos los países se agrupan según sus ingresos atendiendo a las clasificaciones del Banco Mundial: ingreso alto (ingreso nacional bruto per cápita igual o superior a US \$ 9386 en 2003), ingreso medio (US \$ 766 a US \$ 9385) e ingreso bajo (igual o inferior a US \$ 765).

Principales agrupaciones mundiales. Los tres grupos mundiales son: países en desarrollo, Europa Central y Oriental, la Comunidad de Estados Independientes (CEI) y países de la Organización de Cooperación y Desarrollo Económico (OCDE). Estos grupos no son mutuamente excluyentes (de hecho, el reemplazo del grupo de la OCDE por el grupo de países de la OCDE de alto ingreso y la exclusión de la República de Corea sí generaría grupos mutuamente excluyentes).

Clasificaciones regionales. Los países en desarrollo se clasifican a su vez en las siguientes regiones: África Subsahariana, América Latina y el Caribe (incluido México), Asia Meridional, Asia Oriental y el Pacífico, Europa Meridional y Estados Árabes. Estas clasificaciones regionales son congruentes con las Oficinas Regionales del PNUD. Existe una categoría adicional, la de los países menos adelantados, según la definición de las Naciones Unidas (UN-OHRLLS, 2005).

El IDH ha tenido grandes repercusiones en la formulación de políticas de desarrollo: ha estimulado el debate político nacional, ha permitido determinar esferas que requieren atención en las políticas de desarrollo y formulado estrategias concretas, ha permitido abrir nuevos cauces de análisis y ha aportado informes y estadísticas por países. Para conocer más detalles sobre el uso y las restricciones del IDH y los indicadores que lo componen se puede revisar la página http://hdr.undp.org/statistics.

En el año 2003 se realizó el Informe Nacional de Desarrollo Humano específico para Colombia (PNUD, 2003). En la página web http//www.pnud.org.co se pueden consultar diversos documentos e informes sobre el desarrollo humano en Colombia. En la Tabla 1 se relacionan la situación de Colombia en 2005 y las principales metas sociales que se deberían alcanzar de acuerdo con la propuesta VISION 2019 presentada por el primer Gobierno del presidente Uribe. 
Tabla 1. Principales metas sociales que debería alcanzar Colombia.

\begin{tabular}{lcc}
\hline \multicolumn{1}{c}{ (Porcentaje de la población) } & \\
\hline \multicolumn{1}{c}{ Meta } & actual (\%) & $\mathbf{2 0 1 9}(\mathbf{\%})$ \\
\hline Nivel de pobreza & 52.6 & 20.0 \\
\hline Nivel de indigencia & 17.0 & 6.0 \\
\hline Desnutrición infantil & 6.7 & 4.5 \\
\hline Tasa de desempleo (promedio) & 13.6 & 5.0 \\
\hline Cobertura en pensiones & 25.0 & 71.1 \\
\hline Cobertura en educación universitaria & 19.0 & 23.0 \\
\hline Cobertura en educación tecnológica & 6.8 & 17.0 \\
\hline Tasas de deserción infantil & 50.0 & 25.0 \\
\hline Cobertura en salud & 66.0 & 100.0 \\
\hline Cobertura de vacunación & 93.0 & 95.0 \\
\hline Mortalidad infantil * & 24.4 & 14.0 \\
\hline Tasa de fecundidad ** & 2.6 & 1.9 \\
\hline Utilización de métodos anticonceptivos & 64.0 & 78.0 \\
\hline
\end{tabular}

(*) Muertes de niños menores de 1 año por cada 1.000 nacidos vivos.

$(* *)$ Promedio de niños por familia.

Para el Valle del Cauca, muy pronto se tendrá el Informe de Desarrollo Humano-IDH Valle cuyo tema central es la inclusión, y que se está preparando desde Junio de 2006.

\subsection{Cómo lograr la competitividad y el desarrollo}

La competitividad no es un fin en símisma, es una utilidad instrumental o extrínseca relacionada con el tener y es el medio para lograr el desarrollo, el cual es una utilidad no-instrumental o intrinseca relacionada con el ser.

Con frecuencia se persiguen objetivos de crecimiento económico asumiendo que ello conlleva automáticamente el desarrollo social. Sin embargo, no siempre es así, alcanzar niveles satisfactorios de competitividad y desarrollo implica mucho más que conseguir niveles satisfactorios de crecimiento económico (Freeman, 1992). Para Ackoff (1991), el crecimiento es un aumento de tamaño o de cantidad; el crecimiento tiene que ver con propiedades físicas mientras que el desarrollo con propiedades mentales. Se puede crecer sin desarrollarse así como también desarrollarse sin crecer.

Por tanto, al pensar en competitividad se deben considerar sus implicaciones en el desarrollo. La competitividad no es un fin en si misma. El mejoramiento de la posición competitiva de una empresa, de una región o de una nación, debe ir orientado hacia el crecimiento y el desarrollo, no solamente cuantitativo sino cualitativo, es decir, tanto a un incremento en el nivel de vida, representado en un mayor ingreso económico para la personas, como a un incremento de la calidad de vida, representado en un mejor ambiente, una mejor sociedad, una sociedad que se caracterice por el respeto a la libertad, a lo ecológico, a lo cultural, a lo social, a lo demográfico.

De otra parte, vale la pena destacar que la competitividad y el desarrollo no dependen exclusiva y necesariamente de la inserción de la economía de un país en los mercados internacionales. Paul Krugman (1994), connotado economista estadounidense, llama la atención en 
el sentido de que el término competitividad no se puede usar indiscriminadamente tanto para países como para empresas, dado que en principio, por ejemplo, cuando una empresa entra en crisis tiene la posibilidad de cerrar mientras que los países no cierran. Algunos de los dirigentes de naciones, afirma Krugman, pretenden hacer pensar a sus conciudadanos que sus naciones son empresas para hacer un llamado al mejoramiento de la productividad y establecer un balance favorable dentro de la balanza comercial. Las estadísticas también se tienden a acomodar para que contribuyan a aumentar el sofisma de competitividad entre países y tratando de reforzar una clara tendencia de que resultados favorables en comercio exterior aumentan la competitividad en una nación. Krugman cuestiona además el obsesivo interés de competir en el plano del comercio exterior poniendo como ejemplo, que a mediados del siglo pasado los Estados Unidos tenían muy poco comercio exterior y aún así, el estándar de vida de sus ciudadanos era alto, más por la productividad interna de su industria que por el comercio internacional.

\subsubsection{Factores que impulsan la competitividad y el desarrollo de los países}

Michael Porter publicó en 1990 el libro La Ventaja Competitiva de las Naciones con base en la investigación realizada entre los años 1985 y 1990. Después de tanto tiempo, sus resultados están aun vigentes, y son utilizados como un modelo para definir y trazar estrategias de competitividad. Porter trabajó conjuntamente con 10 grupos de investigadores en 10 países desarrollados y líderes en algún sector industrial.

La Figura 1 representa uno de los principales resultados de dicha investigación, en la que se muestran los distintos niveles de desarrollo que pueden ser alcanzados por un país.

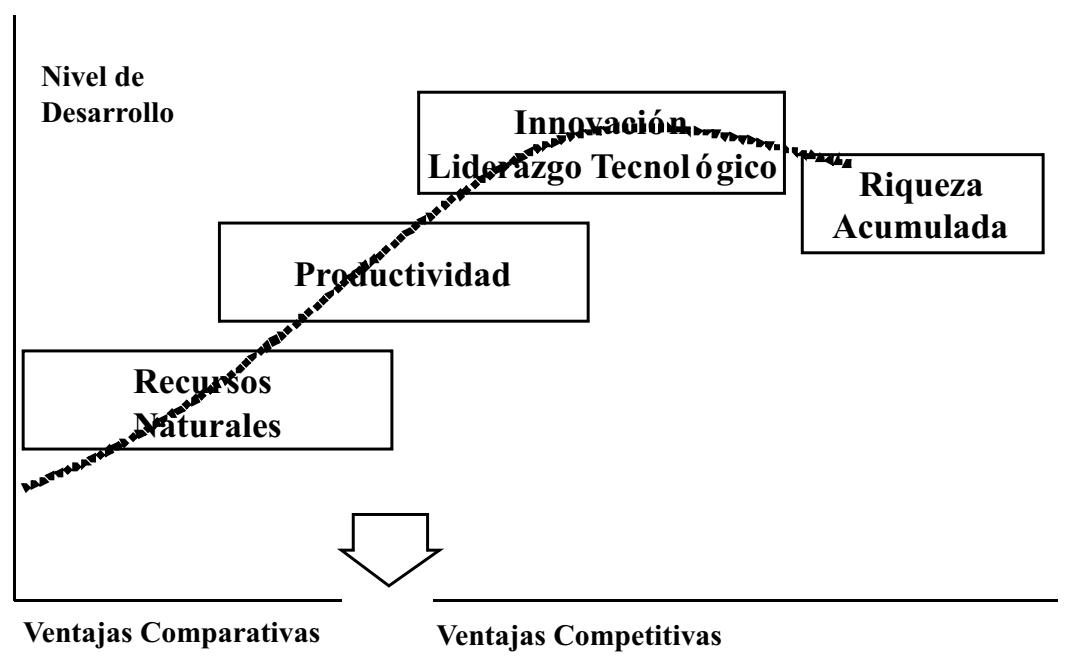

Figura 1. Impulsores del Desarrollo (Adaptado de Porter, 1990). 
Porter clasificó los países en cuatro grupos de acuerdo con su nivel de desarrollo en función de los factores que impulsan dicho desarrollo.

- Nivel de Desarrollo 1. Lo conforman los países que basan su desarrollo fundamentalmente en los recursos naturales. Estos países tienen un nivel de desarrollo relativamente bajo y dentro de estos se encuentra Colombia, cuyo desarrollo se fundamenta en la exportación de petróleo, carbón, café, etc.; se aprovechan los recursos naturales pero en ellos no hay un valor agregado sustancial que permita tener un dinamismo creciente en los ingresos con la exportación de dichos productos.

- Nivel de Desarrollo 2. Lo conforman los países que basan su desarrollo en la productividad, como resultado de la inversión en la automatización de procesos industriales, para lograr mayor valor agregado.

- Nivel de Desarrollo 3. Es el máximo nivel de desarrollo. Lo conforman los países que basan su desarrollo en la innovación, que tienen un liderazgo tecnológico, que están permanentemente implementando nuevos procesos y creando tanto nuevos productos como nuevos negocios y organizaciones, logrando así un mayor valor agregado.

- Nivel de Desarrollo 4. Lo conforman los países que basan su desarrollo en la riqueza acumulada. Como se observa en la figura 1 estos países tienen un nivel de desarrollo inferior a los países que basan su desarrollo en la innovación y en el liderazgo tecnológico, debido a que luego de llegar al nivel de desarrollo máximo, bajaron la guardia, coloquialmente se durmieron en los laureles y como camarón que se duerme se lo lleva la corriente, perdieron jerarquía y nivel de desarrollo.

El nivel de desarrollo más bajo está basado en ventajas comparativas, aquellas que se obtienen al heredar ciertas condiciones naturales, por ejemplo la ubicación geográfica. Se explotan los recursos naturales generando un valor agregado bajo. En contraste, los niveles de desarrollo superiores se basan en ventajas competitivas, logradas mediante la productividad y la innovación; se procesan las materias primas provenientes de los recursos naturales, generando un mayor valor agregado. Las ventajas competitivas son dinámicas y como tal se pueden perder en un determinado momento, porque regularmente los competidores también están ansiosos de copiar y más aun de mejorar lo que los demás están haciendo (máxime si les genera buenos resultados). De ahí que, por esta dinámica, la innovación sea la base de las ventajas competitivas.

“La promesa del mercado no está en los pocos ricos del mundo desarrollado, ni en los consumidores de clase media de los países en desarrollo, sino en los miles de millones de pobres que comienzan a entrar a la economía de mercado".

(C. K. Prahalad)

\subsubsection{Cómo lograr la competitividad}

En los enunciados anteriores se puede constatar que competitividad es un concepto amorfo aún en construcción. Sin embargo, de acuerdo con lo analizado en el parágrafo anterior, las ventajas competitivas se consiguen mediante la productividad y la innovación. Consecuentemente, se puede decir que la competitividad se logra, se conserva y se mejora, en la medida en que se agregue valor a las actividades, procesos, productos y servicios, bien sea mediante la productividad o la innovación. El valor se agrega, tanto bajo la óptica de clientes o usuarios y de la sociedad, como de las organizaciones, empresarios, directivos 0 empleados. Cabe destacar que el origen de la creación de valor está en el cliente, porque es quien finalmente decide entregar una cantidad de dinero a cambio de algo que va a mejorar su funcionalidad, ahorrándole tiempo o algún otro recurso, permitiéndole hacer cosas que antes no podía hacer, etc.

Entonces, ¿qué es productividad y qué es innovación?

Productividad: Capacidad o grado de producción por unidad de trabajo, superficie de tierra cultivada, equipo industrial, etc. Relación 
entre lo producido y los medios empleados, tales como mano de obra, materiales, energía, etc. (RAE, 2001).

El concepto técnico y matemático de productividad es una relación directa entre los productos que se obtienen a la salida de un proceso con la unidad de insumo utilizado. Se habla de productividad laboral, de productividad del capital; en general, de productividad relacionada con cada uno de los distintos recursos utilizados en el proceso. Por ejemplo, en un proceso industrial, si la energía juega un papel importante, un indicador de productividad clave es la cantidad de productos que se obtienen a la salida de dicho proceso en relación con cada $\mathrm{kW}$-h de energía que se consume. En un almacén de ventas de productos de consumo masivo, un indicador importante de productividad es la cantidad de dinero vendida por la unidad de metro cuadrado utilizada para la exposición de sus productos.

Cuando se habla de productividad laboral, es conveniente medirla en términos del número de productos que se obtienen en relación con las horas-hombre trabajadas. Cabe destacar que con frecuencia no se utilizan las horas-hombre trabajadas, sino el número de personas que trabajan, lo cual puede conducir a errores puesto que podría ser que dichas personas trabajen turnos de 8,10 ó más horas.

Sin embargo, el concepto de productividad va más allá de la simple consideración cuantitativa presente en la relación productos por unidad de insumo (Drucker, 1991). El Centro de Productividad del Japón y todos los centros nacionales de productividad del Asia Pacífico adoptaron como filosofía de trabajo, el siguiente concepto que enunció en París la Agencia Europea para la Productividad en 1959:

Productividad es ante todo un estado de la mente. Es una actitud que busca el mejoramiento continuo de todo cuanto existe. Es la convicción de que las cosas se pueden hacer hoy mejor que ayer y que mañana se podrán hacer mejor que hoy. Adicionalmente, significa un esfuerzo continuo para adaptar las actividades económicas a situaciones que cambian permanentemente y la aplicación de teorías y métodos nuevos. Es un convencimiento firme en el progreso de la humanidad.

Múltiples ejemplos corroboran que, en determinados momentos, las personas hacen más de lo que estaba previsto, superan con creces el estándar que estaba definido, básicamente por efecto de un estado de motivación para utilizar mejor los recursos disponibles.

Los japoneses adoptaron el concepto de productividad como una actitud de mejoramiento continuo frente a la vida, como una manera de vivir, no solo como una filosofía de trabajo; realmente, no es fácil ser productivo en el trabajo y no serlo en la cotidianidad de la vida.

El concepto de productividad presentado, que se remonta a 1959, habla de "un esfuerzo continuo para adaptar las actividades económicas a situaciones que cambian permanentemente y la aplicación de teorías y métodos nuevos". Se trata de ser flexibles, de ser innovadores. Es decir, la innovación esta involucrada en este concepto de productividad.

Finalmente, el concepto de productividad habla de "un convencimiento firme en el progreso de la humanidad". Es decir, la competitividad no tiene sentido en si misma, el sentido lo da el progreso de la humanidad. Productividad e innovación tienen que ir en pro del desarrollo integral (social, cultural, económico, ecológico, etc.) de la humanidad.

Innovación: Creación o modificación de un producto, y su introducción en un mercado. (RAE, 2001).

En relación con la innovación (Drucker, 1986; Hamel et al., 1995; Freeman, 1999) puede decirse que innovación es la conversión de conocimiento en nuevos productos o procesos para la explotación exitosa de un mercado.

Por tanto, la creación de nuevas organizaciones o negocios está asociada con la innovación. Son las empresas las que innovan, aunque otros agentes pueden facilitar o acelerar el proceso. 
Es un factor clave para la competitividad en mercados abiertos. Las empresas que no innovan sólo pueden sobrevivir a mediano plazo en mercados protegidos. Un concepto más pragmático es el siguiente:

Innovar consiste en convertir en oportunidades los problemas, las carencias, y las amenazas; la innovación surge de la creatividad y esta, a su vez, se nutre de las restricciones y de las necesidades.

Se destacan tres posturas para asumir frente a la innovación tecnológica:

- Líderes: son los que se toman menos tiempo para introducir innovaciones en el mercado y generalmente logran sacar gran parte de los beneficios que conlleva el mayor valor agregado creado a través de la innovación. Tienen una actitud innovadora proactiva y desarrollan una estrategia tecnológica de innovación sistemática y permanente, a tal punto que ellos mismos sacan del mercado sus propios productos y servicios haciéndolos obsoletos. Tienen muy claro que el valor es definido por el cliente, que el valor es sensible al tiempo y al contexto, que no puede ser definido ni creado por una sola área funcional de la organización y que la empresa colabora con otras organizaciones para satisfacer total o parcialmente las necesidades del cliente.

- Seguidores: son los que en un determinado momento copian lo que han desarrollado los líderes pero tardan un periodo de tiempo para lograr imitarlos.

- Rezagados: son los que tardan mucho más tiempo para introducir los cambios, en sus procesos o en sus productos y en consecuencia deben conformarse con los 'sobrados' que dejan los líderes y los seguidores.

\subsubsection{Cómo lograr el desarrollo}

De acuerdo con la definición y los conceptos analizados anteriormente, puede decirse que el desarrollo se logra mediante la evolución progresiva de la satisfacción de las necesidades propias legítimas y las de los demás.
Para lograr dicha evolución progresiva, vale la pena tener presente reflexiones de Max-Neef y de Prahalad. A Max-Neef $(1986,1993)$, cuando dice que las necesidades humanas fundamentales son finitas, pocas y clasificables, y que son las mismas en todas las culturas y en todos los períodos históricos. Lo que cambia, a través del tiempo y de las culturas, es la manera o los medios utilizados para satisfacerlas. A Prahalad (2005), cuando sostiene que la transformación social y económica puede ser muy rápida cuando los consumidores de la base de la pirámide (los 4 mil millones de personas que tienen un ingreso inferior a USD 1500/Año; en Colombia alrededor de 20 millones) tengan la oportunidad de participar y beneficiarse de elegir productos y servicios que se ponen a su disposición mediante mecanismos de mercado. Para ello, siguiendo a Prahalad, se requiere dejar de pensar en los pobres como víctimas o como carga y reconocerlos como empresarios creativos lo que conducirá a la apertura de un mundo de nuevas oportunidades.

Este planteamiento de cómo lograr el desarrollo está respaldado por los principios que formuló el economista austriaco Joseph Schumpeter (1942), denominados de la 'destrucción creativa', que consisten simplemente en reestructurar constantemente la empresa con el fin de, por una parte, lograr un mejor rendimiento de la misma en el mercado; y, por la otra, poder hacer frente a la competencia y mantener el liderazgo en la industria.

Schumpeter planteó sus posiciones sobre el capitalismo como un sistema evolutivo y la mutación, sobre los emprendedores y la innovación. La marca indeleble de su pensamiento es la lograda expresión y explicación de la 'destrucción creadora': el "proceso de destrucción creadora constituye el dato de hecho esencial del capitalismo. En ella consiste en definitiva el capitalismo y toda empresa capitalista tiene que amoldarse a ella para vivir". Es un proceso también llamado de "mutación industrial' que revoluciona incesantemente la estructura económica desde dentro, "destruyendo ininterrumpidamente lo antiguo y creando continuamente elementos nuevos". 
Schumpeter observa el "vendaval perenne de la destrucción creadora" en la apertura de nuevos mercados, en los nuevos bienes de consumo, en una nueva materia prima, en los nuevos métodos de producción y de transporte que crea la empresa capitalista. Para él, este proceso es el "impulso fundamental que pone y mantiene en movimiento a la máquina capitalista" y el 'progreso técnico' capitalista es la destrucción de capital en los niveles donde penetra la competencia de la nueva mercancía o el nuevo método de producción.

Para Schumpeter, la empresa y el progreso técnico capitalista son "esencialmente una y la misma cosa", o, dicho de otra manera, "el primero ha sido la fuerza propulsora del segundo".

\subsection{Algunos planteamientos críticos frente a la teoría porteriana de la competitividad}

La competitividad es uno de los temas más tratados por la literatura económica de los últimos años. Las economías nacionales están enfrentadas en una guerra industrial, económica y tecnológica, cada una guiada por el objetivo de ser más fuerte que el resto de los países. Sin duda alguna, quien más ha influido en el predominio de esta concepción es Michael Porter, quien puede ser considerado el Keynes de la estrategia competitiva. Sin embargo, el tema está lejos de haber sido agotado y permanentemente nuevas contribuciones al análisis de la realidad abren otras perspectivas y líneas de investigación.

A continuación se reseñan algunos planteamientos críticos que llaman la atención y provocan el debate sobre diferentes aspectos del modelo de Porter, y que es necesario tomar en cuenta para complementar el marco de análisis presentado.

David Yoffie, profesor de la Escuela de Negocios de Harvard, al igual que Porter, fue uno de los primeros en señalar las limitaciones del modelo porteriano para explicar los intercambios de bienes y servicios que efectivamente se producen en el mundo real. En resumen, el aporte de Yoffie (1993) consiste en destacar que los mercados oligopólicos y las prácticas proteccionistas adoptadas principalmente por los países industrializados determinan un contexto marcadamente distinto del modelo planteado por
Porter, y que se debe tener en cuenta que en la realidad el comercio internacional no depende sólo de las ventajas competitivas de las naciones.

Para Paul Krugman (1994), la noción de que el crecimiento económico de los países está determinado por su éxito en los mercados mundiales no es necesariamente cierta. Este autor afirma que identificar competitividad con crecimiento no sólo es erróneo, sino que también puede resultar peligroso para el sistema económico.

- Los países tienen que ser productivos para generar más valor, independientemente de sus competidores internacionales; lo importante es la productividad total de los factores, no importa el mercado para el que se produce (local o internacional).

- Los sectores de alto valor agregado no son mejores negocios.

- Pensar en términos de competitividad puede acarrear varios peligros, entre ellos, desperdiciar recursos gubernamentales supuestamente dirigidos a mejorar la competitividad, provocar el proteccionismo o guerras comerciales y generar la aplicación de inadecuadas políticas económicas respecto a aspectos fundamentales.

\subsection{Situación que actualmente afrontan Colombia y América Latina}

Algunas de las características que actualmente afrontan los países en desarrollo son:

- Competir por la localización de la producción y la inversión extranjera, donde las multinacionales tienen cada vez más poder, por lo que la soberanía de los estados se hace vulnerable a los deseos de las multinacionales.

- Entrar en el sistema de competencia global. De allí buena parte de su energía la han dedicado a desmontar los viejos modelos de desarrollo y por supuesto, como esos planes se basaban en la acción del estado paternalista, entonces lo prioritario ha sido su reestructuración $\mathrm{y}$ modernización. 
- Millones de medianos, pequeños y microproductores encuentran que el mercado en el cual sus capacidades productivas eran pertinentes ha desaparecido. Producían y comercializaban en y para mercados locales que fueron destruidos o mejor, expropiados.

- Lo que era competitivo en un mercado regional o nacional no está resultando serlo en el nuevo mercado globalizado, lo cual está implicando la destrucción masiva de las capacidades productivas que se encuentran en manos de la gran mayoría de los productores y trabajadores de los países menos avanzados.

Por lo tanto, las condiciones en toda América Latina no son muy favorables, y son pocas las empresas que logran insertarse adecuadamente en la dinámica de crecimiento, configurando pocos grupos empresariales con perfil ganador, generalmente con un perfil familiar, que conservan el liderazgo de las industrias que operan en los países y representan el núcleo de los grandes grupos industriales $y$ financieros nacionales.

Frente a esta situación, el premio Nóbel de Economía Joseph Stiglitz (2003) pide planes de crecimiento más inteligentes y critica al Fondo Monetario Internacional (FMI). Algunos apartes de sus reflexiones son:

"Colombia sí puede tener una estrategia de crecimiento. Es más, es necesario que tenga una estrategia propia. El hecho de que éste es un pais dificil, que no puede tener una politica fiscal expansionista hace que el diseño de una estrategia de crecimiento sea dificil, lo que significa que se debe hacer énfasis en politicas estructurales, lo que a su vez significa que hay que poner más énfasis en proveer créditos a las medianas y pequeñas empresas. Quiero decir que la estrategia de crecimiento debe ser más inteligente. Ello se logra concentrándose en las exportaciones, teniendo una estrategia seria para promoverlas y también a través de créditos. Entonces, a largo plazo se tiene que concentrar en la educación y la tecnología. Hay tantas cosas que se pueden hacer. Pero si es tan posible y viable, ¿por qué no se ha hecho? Pienso que parte del problema es la influencia del Fondo Monetario
Internacional y de las políticas del llamado consenso de Washington, que expusieron la idea de que una vez se logre la estabilización, la privatización y la liberalización, el crecimiento llegaría por sí solo. Según esto, no se necesitaría de una estrategia de crecimiento porque los mercados se encargarían de eso.

Como ejemplo de economías en el mundo que demuestran la efectividad de mis puntos de vista están los países del este asiático; todos crecieron como resultado de una estrategia de crecimiento explícita. Todos ellos rechazaron los estándares de las políticas del consenso de Washington. Ellos reconocieron que la estabilización no era suficiente. Es más, en muchos de estos países fueron creadas empresas públicas para promover el crecimiento. Las acerías de Corea del Sur y Taiwán han sido de las más exitosas del mundo y ayudaron a sus países a crecer rápidamente. Hace 40 años, Corea era un país agrícola pobre, hoy por hoy es quizás el segundo exportador de circuitos integrados de computador y es la decimosegunda economía del mundo.

Todo esto, fue logrado porque simplemente se tuvo una estrategia de crecimiento explícita.

Parte de los problemas que Colombia enfrenta hoy es que atravesó por una fuerte recesión hace dos años. Como resultado de ello y de la sobrevaloración de las casas de cambio y altos intereses, muchas firmas quebraron, perdieron la confianza. Toma mucho tiempo la creación de nuevas compañias. Cuando una economía es recesiva, es muy complicado encontrar inversionistas que tomen el riesgo de invertir en estos países. Entonces, el fracaso más destacado en toda economía de mercado es el fracaso de mantener la macroeconomía en armonía con el crecimiento económico."

\section{Ingeniería}

Ingeniería: Estudio y aplicación, por especialistas, de las diversas ramas de la tecnología (RAE, 2001).

Ingeniería genética: Tecnología de la manipulación y transferencia del ADN de unos organismos a otros, que posibilita la creación de nuevas especies, la corrección de defectos génicos 
y la fabricación de numerosos compuestos útiles (RAE, 2001).

Tecnología: Conjunto de teorías y de técnicas que permiten el aprovechamiento práctico del conocimiento cientifico (RAE, 2001).

Ingenio (del latin, ingenŭum) (RAE, 2001):

- Facultad del hombre para discurrir o inventar con prontitud y facilidad.

- Intuición, entendimiento, facultades poéticas y creadoras.

Se destaca que el concepto de ingeniería está estrechamente ligado tanto al concepto de tecnología como al de ingenio; a su vez, este último lo está con el de innovación. La tecnología, en su concepción más sencilla, consiste en conocimiento aplicado, el cual utilizado estratégicamente conduce a un incremento en la creación de riqueza, bien sea por productividad, resultado de aplicar conocimiento a tareas conocidas, o por innovación, resultado de aplicar conocimiento a tareas nuevas (Drucker, 1986).

La ingeniería, entonces, juega un papel crucial en las decisiones que tienen que ver con la sociedad, más aún, en virtud de las nuevas tecnologías y del acelerado desarrollo o avance tecnológico. Decisiones representadas en la aplicación del conocimiento, tanto científico como empírico, para desarrollar las formas en que se pueden utilizar, de manera económica y sostenible, los materiales y las fuerzas de la naturaleza, con el fin de obtener para un determinado problema, de entre las posibles soluciones, aquella que funcione de manera más satisfactoria en beneficio de la comunidad. Conocimiento que se adquiere a través del estudio, la experiencia y la práctica de las ciencias matemáticas y naturales y que se aplica mediante la toma de decisiones en ambiente de incertidumbre, con un conjunto de datos, no necesariamente completos ni exactos.

Cabe destacar, que no todos los problemas son complejos, ni mucho menos sus soluciones (Ohmae, 1992; Mintzberg et al., 1997; Eisenhardt \& Sull, 2001). Por tanto, la ingeniería, en lo posible, debe proponer e implementar soluciones prácticas, no necesariamente complejas, mediante tecnologías, lo más simples y de bajo costo posible, para tratar problemas específicos.

“ La sencillez es lo más fácil del mundo. Es el último término de la experiencia y el último fruto delgenio".

(George Sand)

\section{Conclusiones}

A continuación, a manera de conclusiones, se presentan algunas reflexiones, enfatizando en la importancia del papel que juega la ingeniería en el desarrollo y la competitividad.

- La productividad y la competitividad no son un fin en si mismas, su mejoramiento no conduce automáticamente a un mejoramiento de lo social y de lo económico. Se debe aprender de los hechos aun de otros países, tales como Japón, que en 1955 creó el Japan Productivity Center, buscando fundamentalmente el mejoramiento de la productividad. Posteriormente, en 1973, casi 20 años después, creó otra entidad de ámbito nacional, el Social and Economic Development Congress (SEDC), con el objetivo de buscar su desarrollo socioeconómico. En 1994, casi 40 años después de crear el JPC, se fusionaron estos dos entes conformando uno nuevo el JPC-SEDC, buscando generar un ambiente de productividad orientado hacia el desarrollo social y económico de la comunidad. Los japoneses entendieron que la productividad no era un fin en sí misma y que no era conveniente tener un centro en pro del mejoramiento de la productividad y por otro lado tener otro centro buscando el mejoramiento del bienestar, lo social y económico.

- Las ventajas naturales ceden terreno ante factores de avanzada, como el aprendizaje, la capacitación, la infraestructura de transporte y comunicaciones, la ciencia y la tecnología. La economía de los países depende cada vez más de las ventajas creadas por su gente y relativamente menos de las ventajas heredadas de la naturaleza, aún cuando ambas estén, desde luego, asociadas. La posición geográfica, el clima y las materias primas, pasan definitivamente a un plano inferior (Porter, 1991). No basta con poseer los mejores recursos, sino saber utilizarlos estratégicamente. En un mercado libre y globalizado como el actual, 
sólo pueden sostenerse las ventajas competitivas generadas por creaciones intelectuales (Nomen, 2005). Se deduce que las ventajas comparativas, 'per se', de países como Colombia, no son suficientes para obtener ventajas competitivas, no basta con poseer recursos, sino saber utilizarlos estratégicamente.

- La tecnología y el conocimiento son entonces un factor clave para la creación de riqueza y el mejoramiento de la competitividad; juegan un papel crucial hoy en día en la globalización y su gestión estratégica es la base para lograr incrementar la productividad, generar innovación $\mathrm{y}$ crear nuevos negocios en las organizaciones.

Producir eficientemente se logra incorporando tecnología aplicada a los procesos de producción. La transferencia de tecnología es un elemento crucial que interviene en este proceso, asegurando flujos de conocimiento y capacitación para pasar a una fase intermedia en la que la eficiencia en la producción más la reducción de costos y las mejoras en calidad, diseño, materiales, empaque, comercialización, gerencia, etc., hacen más productiva a una región. Mediante la combinación de mayor productividad y menores costos de producción se obtiene en la tercera fase un nivel suficiente de competitividad.

-El análisis del papel que desempeña la ingeniería en la competitividad y el desarrollo se hace mucho más complejo en virtud de las regulaciones impuestas por los países más desarrollados (PMD) a los países menos desarrollados (PmD). Uno de los efectos del nuevo orden económico internacional, de la globalización, ha sido restarle importancia a las ventajas comparativas que poseen los $\mathrm{PmD}$, debido a los bajos precios internacionales de los productos naturales que exportan. Con raras excepciones, como en el caso del petróleo, los precios de los recursos naturales, provenientes de los $\mathrm{PmD}$, que se venden en el mercado internacional, sufren año a año una depreciación en términos de poder adquisitivo, frente a los productos finales, fruto de su transformación en los procesos industriales de los PMD. Lo cual corrobora que cada vez sean más importantes las ventajas competitivas y que pierdan peso las ventajas comparativas (PérezCastaño, 2005).
Es por ello que, con frecuencia, un producto natural con bajo valor agregado tecnológico, paradójicamente, tiene en el mercado internacional un precio de venta menor si proviene de un PMD que de un PmD, en virtud del efecto de los subsidios gubernamentales que conceden los gobiernos de los PMD a sus agricultores. Por ejemplo, el maíz, el trigo o el algodón norteamericano tienen en el mercado internacional un precio mucho menor que el colombiano, a pesar de que Colombia cuenta con tierra más productiva y mano de obra mucho más barata que la estadounidense. No es que el costo de producción del maíz, trigo o algodón norteamericano sea mucho menor que el colombiano; por el contrario es sustancialmente mayor. La explicación está en el subsidio que el gobierno estadounidense le concede a esos productos.

Los países menos desarrollados tienen que ser muy sagaces, osados e innovadores en la aplicación estratégica de la ingeniería para afrontar las regulaciones que les imponen los países más desarrollados.

Por ejemplo, incrementar la baja producción agrícola con semillas mejoradas y tecnologías para la renovación del suelo y la administración del agua; tratar las enfermedades diarreicas, con tecnologías apropiadas para agua potable. Considerar que los teléfonos celulares e Internet, y no sólo un mayor número de vías pavimentadas, contribuyen a romper el aislamiento de muchas veredas colombianas.

- La industrialización es la medida común del desarrollo económico, pero no existe una razón por la cual la agricultura no pueda sustentar una economía (The Economist, 1998). Por lo tanto, un reto para la ingeniería colombiana puede ser llevar la tecnología al campo para agregar valor a los productos agrícolas. Aprovechar todos y cada uno de los campos del conocimiento de la ingeniería: de alimentos, mecánica, industrial, etc., para procesar in situ las frutas, verduras y hortalizas, de tal manera que se pueda transformar, por ejemplo, un bulto de limones en un pequeño recipiente con zumo puro de limón y este a su vez en un reducido frasco con extracto de zumo de limón. Como, lo más probable es que no haya energía eléctrica 
disponible in situ, entonces se requerirá del concurso de la ingeniería eléctrica y electrónica para desarrollar sistemas de generación de energía aprovechando los recursos naturales disponibles, agua, viento, sol, etc.. En fin, se debe analizar (con visión holística) la situación para que cada rama de la ingeniería dé el aporte que le corresponde. Muy seguramente, se obtendrán beneficios tangibles en lo económico e intangibles en lo social.

Beneficios económicos, tales como un mayor aprovechamiento de los productos cosechados, un mayor valor agregado a los productos, una mejor remuneración para los trabajadores del campo, etc. Beneficios sociales, tales como mejores condiciones de vida para los campesinos, disminución de la migración de personas del sector rural al sector urbano de las grandes ciudades, etc.

- En esencia, desarrollar capacidades para aprovechar estratégicamente los recursos con que cuentan las regiones (Grant, 1991). Dado que la competitividad no es un fin en sí misma sino el medio para lograr el desarrollo, la ingeniería debe contribuir al desarrollo, ampliando las oportunidades de las personas, mediante la expansión de sus capacidades para llevar el tipo de vida que valoran y que tienen razones para valorar. Esto es, a través de la formación de capacidades humanas y su utilización para satisfacer tanto las necesidades existenciales -lo que se requiere para existir o estar en el mundo- como las necesidades esenciales -lo que le permite al ser humano llegar a ser lo que potencialmente es.

La ingeniería contribuye a la creación de riqueza, al desarrollo y a la competitividad apoyando la obtención de mayor productividad mediante la aplicación de tecnologías a las tareas existentes, a lo cotidiano; apoyando la innovación, mediante el desarrollo de nuevas tecnologías para implementar nuevos procesos, introducir nuevos productos en el mercado, crear nuevos negocios, empresas u organizaciones.

- Finalmente, la invitación es a practicar la ingeniería con inteligencia, intuición e imaginación para resolver los problemas de la región, recordando a Ortega y Gasset cuando dice que la mayor parte de los hombres tienen una capacidad intelectual muy superior al ejercicio que hacen de ella.

Ingeniería, inteligencia, intuición e imaginación: Se requiere de un ejercicio inteligente, intuitivo e imaginativo de la ingeniería para resolver los problemas de la región y la sociedad.

\section{Agradecimientos}

El autor desea manifestar explícitamente sus sentimientos de gratitud con la Universidad del Valle, Colombia y la Universidad Politécnica de Valencia (UPV), España, por el apoyo que ha recibido para desarrollar investigaciones que le han permitido plasmar estas reflexiones.

El autor agradece los aportes de Juan Ignacio Dalmau Porta, catedrático de la UPV y de los evaluadores del artículo, cuyas sugerencias fueron muy pertinentes y apropiadas para concretar algunos planteamientos. De manera especial, el autor agradece al editor jefe de la revista Ingeniería y Competitividad por su invitación a escribir este artículo.

\section{Referencias bibliográficas}

Ackoff, R. L. (1991). Ackoff's fables. Irreverent reflections on business and bureaucracy. New York: John Wiley \& Sons, Inc.

Ackoff, R. L., \& Roven, S. (2003). Redesigning society. California: Stanford University Press.

Drucker, P. F. (1986). La innovación y el empresario innovador. Bogotá: Editorial Norma.

Drucker, P. F. (1991). The new productivity challenge. Harvard Business Review 69 (6), 69-80.

Drucker, P. F. (1992). Gerencia para el Futuro. Bogotá: Editorial Norma.

Drucker, P. F. (1994). La sociedad postcapitalista. Bogotá: Editorial Norma.

Eisenhardt, K. M., \& Sull, D. (2001). Strategy as simple rules. Harvard Business Review 79 (1), 107-116. 
Freeman, C. (1992). The economics of hope: essays in technical change, economic growth, and the environment. London: Pinter Publishers.

Freeman, C. (1999). The economics of industrial innovation. London: Frances Pinter.

Grant, R. M. (1991). The resource-based theory of competitive advantage: implications for strategy formulation, California Management Review $33(3), 114-135$.

Hamel, G., \& Prahalad, C.K. (1995). Compitiendo por el futuro. Barcelona: Editorial Ariel.

Haq, M.-u. (1995). The human development paradigm. In: S. Fukuda-Parr \& A. K. Shiva Kumar (editors), Reflections on human development: concepts, measures and policies for a development paradigm. United Kingdom: Oxford University Press, Chapter I, Section 2. (2004).

IMD (Institute for Management Development). (1996). World Competitiveness Yearbook.

Krugman, P. (1994). Competitiveness: a dangerous obsession. Foreign Affairs 73 (2).

Landau, R. (1991). How competitiveness can be achieved: fostering economic growth and productivity. In: Technology \& Economics (National Academy of Engineering), Washington, D.C. : National Academy Press.

Max-Neef, M. (1993). Desarrollo a escala humana: conceptos, aplicaciones y reflexiones. Barcelona: Editorial Icaria.

Max-Neef, M., Elizalde, A., \& Hopenhayn, M. (1986). Desarrollo a escala humana: una opción para el futuro. En: Development Dialogue, $\mathrm{N}^{\circ}$ especial. Cepaur \& Fundación Dag Hammarskjöld,p. 9-93.

MINCOMERCIO (Ministerio de Industria, Comercio y Turismo de Colombia). (2005). Reporte global de competitividad 2005-2006, World Economic Forum.

http://www.mincomercio.gov.co/eContent/News Detail.asp?ID=3264\&IDCompany $=1$
MINCOMERCIO (Ministerio de Industria, Comercio y Turismo de Colombia). (2006). Informe sobre el anuario mundial de competitividad 2006, Instituto Internacional para el Desarrollo Gerencial (IMD).

http://www.mincomercio.gov.co/econtent/Docu mentos/competitividad/2006/Indicadores/Inform eIMC2006.pdf

Mintzberg, H., Quinn, J. B., \& Voyer, J. (1997). El proceso estratégico. Editorial Prentice Hall Latinoamericana.

Monitor Company. (1994). Informe sobre el Estudio de Competitividad de Colombia.

Monitor Company. (1996). El Valle del Cauca de cara hacia el futuro. Cámara de Comercio de Cali.

Morris, T. (1997). Si Aristóteles dirigiera General Motors: las enseñanzas clásicas para vivir en un mundo competitivo. Bogotá: Editorial Planeta.

Nomen, E. (2005). ; Por fin cae el monopolio tecnológico en $\mathrm{I}+\mathrm{D}+\mathrm{i}$ !. Diario Expansión (diciembre 5, 2005).

Ohmae, K. (2005). La mente del estratega. El triunfo de los japoneses en el mundo de los negocios. Bogotá: Editorial Norma.

Pérez-Castaño, B.J. (2004). Competitividad empresarial. Cali: Editorial Artes Gráficas del Valle.

Pérez-Castaño, B.J. (2005). Tecnología y conocimiento para la competitividad empresarial. En: J. Medina-Vásquez \& G. Rincón-Bergman, La prospectiva tecnológica industrial. Contexto, fundamentos y aplicaciones. Cali: Universidad del Valle, Programa Editorial.

PNUD (2003). El conflicto, callejón con salida. Informe Nacional de Desarrollo Humano -2003. http://indh.pnud.org.co/informe2003_plx?pga= CO3tablaContenido\& $\mathrm{f}=1157407052$ 
PNUD (2005). Informe sobre el Desarrollo Humano 2005. La cooperación internacional ante una encrucijada: ayuda al desarrollo, comercio y seguridad en un mundo desigual.

http:/hdr.undp.org/reports/global/2005/espanol/

Porter, M.E. (1982). Estrategia competitiva. Mexico: Editorial CECSA.

Porter, M.E. (1983). The technological dimension of competitive strategy. In: J. Rosenbloom (editor), Research on technological innovation, management and policy, Vol.1, p.1-33.

Porter, M.E. (1985). Technology and competitive advantage. The Journal of Business Strategy $5(3), 46-64$.

Porter, M.E. (1988). Ventaja competitiva. Mexico: Editorial CECSA.

Porter, M.E. (1990). La ventaja competitiva de las naciones. Mexico: Editorial CECSA.

Porter, M.E. (1991). La ventaja competitiva de las naciones. Buenos Aires: Editorial Vergara.

Prahalad, C.K. (2005). La oportunidad de negocios en la base de la pirámide. Bogotá: Editorial Norma.

RAE (Real Academia Española). (2001). Diccionario de la lengua española. Edición 22. España: Editorial ESPASA. http://www.rae.es/

Rodríguez-Vargas, J.J. (2005). La nueva fase de desarrollo económico y social del capitalismo mundial. Tesis de doctorado, Facultad de Economía, División de Estudios de Posgrado, Universidad Nacional Autónoma de México. http://www.eumed.net/tesis/jjrv/

Schumpeter, J. (1952). Capitalismo, Socialismo y Democracia. Mexico: Editorial Aguilar.

Sen, A.K. (1973). On economic inequality. Oxford, United Kingdom: Clarendon Press.

Sen, A.K. (1976). Poverty and economic development. Second Vikram Sarabhai Memorial Lecture, Vikram A. Sarabhai AMA Memorial Trust, Ahmedabad, India.
Sen, A.K. (2000). Desarrollo y libertad. Barcelona: Editorial Planeta.

Stiglitz, J. (2003). Hacia una economía sustentable: conflicto y postconflicto en Colombia. Conferencia dictada en Bogotá, marzo 9 de 2003.

The Economist (1998) ¿Perdidos en el campo? The Economist, London. (Traducción al español publicada en la revista Suma, septiembre de 1998).

UN-OHRLLS (United Nations Office of the High Representative for the Least Developed Countries, Landlocked Developing Countries and Small Island Developing States). (2005). Indicadores del desarrollo humano, p. 237.

http://hdr.undp.org/reports/global/2005/espanol/p df/HDR05_sp_HDI.pdf

UNDP (United Nations Development Programme). (1990). Human Development Report 1990: concept and measurement of human development.

http://hdr.undp.org/reports/view_reports.cfm?yea $\mathrm{r}=1990$ \& country $=0 \&$ region $=0 \&$ type $=0 \&$ theme $=$ $0 \&$ launched $=0$

UNDP (United Nations Development Programme). (2005a). Human development index rank.

http://hdr.undp.org/docs/statistics/indices/hdi_20 04.pdf

UNDP (United Nations Development Programme). (2005b). Human Development Report 2005.

http://hdr.undp.org/statistics/data/indicators.cfm? $\mathrm{x}=133 \& \mathrm{y}=1 \& \mathrm{z}=1$

WEF (World Economic Forum). (2006). www.weforum.org/en/index.htm

Yoffie, D. B. (1993). Introduction: from comparative advantage to regulated competition. In: D. B. Yoffie (editor), Beyond free trade: firms. governments, and global competition. Boston: Harvard Business School Press. 